\title{
ON THE CONCEPT OF PERIAPSIS IN HILL'S PROBLEM
}

\author{
B.F. VILLAC* and D.J. SCHEERES \\ Department of Aerospace Engineering, The University of Michigan, Ann Arbor, MI, USA, \\ e-mails: bvillac@caltech.edu, scheeres@umich.edu
}

(Received: 4 November 2003; revised: 7 May 2004; accepted 22 May 2004)

\begin{abstract}
The concept of closest approach is analyzed in Hill's problem, resulting in a partitioning of the position space. The different behavior between the direct and retrograde motion is explained analytically, resulting in a simple estimate of the variation of Hill's periodic and quasi-circular orbits as a function of the Jacobi constant. The local behavior of the orbits on the zero velocity surfaces and an analytical definition of local escape and capture in Hill's problem are also given.
\end{abstract}

Key words: Capture, escape, Hill's problem, periapsis, periodic orbits

\section{Introduction}

Circular orbits in a two body problem can be viewed from three different perspectives. The most obvious one is to consider circular orbits as particular periodic solutions. On a different prospective, circular orbits can be considered as delineating the separation between periapsis and apoapsis regions. Indeed, we know that at a fixed energy, periapsis passages of a non-circular motion must be located inside the sphere $\mathcal{S}$ formed by the circular orbits (having the given energy). Moreover, for elliptic motion (i.e., for negative energies), apoapsis are located outside this sphere $\mathcal{S}$ but inside the sphere $\mathcal{Z}$ formed by the zero velocity surface (which corresponds to the sphere of radius twice that of $\mathcal{S}$, as shown on Figure 1). This last remark brings us to the third viewpoint: circular orbits as scaling of the zero velocity surface.

As we know since Poincaré, periodic orbits can be continued into the three body type models (circular restricted three body problem and Hill's problem). In this paper, we show that the two other points of view on circular orbits (based on the periapsis notion) continue to exist in the three body type models, even though the three points of view now result in three different objects. In particular, the second viewpoint results in surfaces that partition the position space in the same way as in the two body problem, except that the direction of motion (direct or retrograde) does matter in this case. The analysis yields also some estimates of the separation between these type of

${ }^{*}$ Current address: California Institute of Technology, Pasadena, CA, USA. 


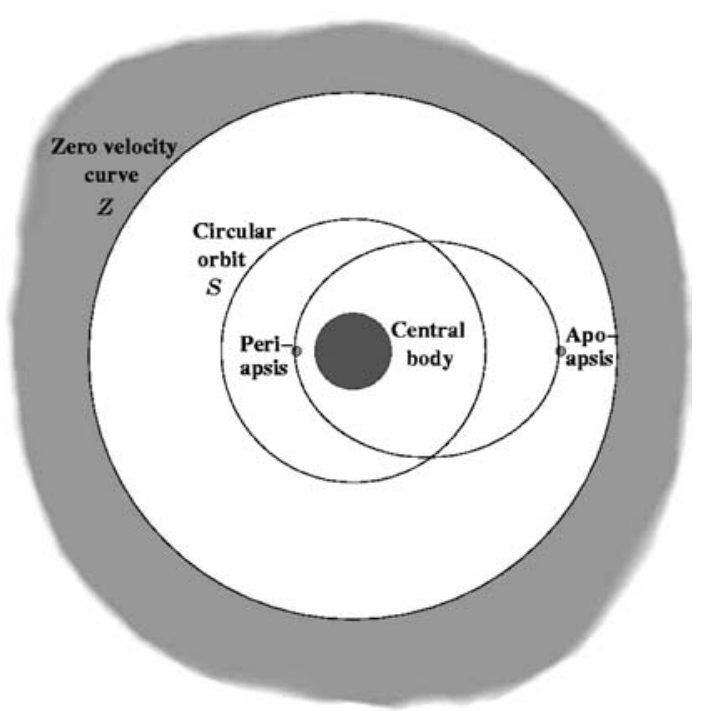

Figure 1. The role of circular orbits in Kepler's problem.

motion and the variation of Hill's periodic orbits as a function of the Jacobi constant. Further qualitative results are also exhibited, notably a suggested definition for local, low energy escape and capture in Hill's problem.

\section{Hill's Problem and Definition of Periapsis}

Hill's problem, first derived by Hill (1878) to analyze the motion of the Moon, models the relative motion of two small masses, gravitationally interacting with each other, and perturbed by a large, distant body. As such, it is a different approximation of the three body problem from the circular restricted three body problem (CR3BP) (see, for e.g., Hénon and Petit (1986) and Villac (2003) for further discussions of this point), even though both approximations share a common realm of application: the motion of a massless particle around a small mass as perturbed by a large body (see Szebehely (1967) and Hénon (1969) for a discussion of this point of view).

\subsection{HILL'S PROBLEM}

Denoting $\mu$ as the sum of the gravitational parameters of the two small bodies and assuming the center of mass of these two small masses, $\boldsymbol{c}$, to be close to a circular motion around the perturbing body, the motion of $c$ and 
the relative motion of the small masses, $\boldsymbol{q}$, decouple to the first order in $\mu^{1 / 3}$. That is, one can study, in a first approximation, the relative motion of the two small masses independently from the motion of their center of mass.

These relative dynamics are described by Hill's equations of motion, which are more easily formulated in a frame following the mean center of mass of the two small masses ${ }^{1}, \bar{c}$, and rotating with the constant, mean angular velocity, $\omega$ (see Hénon and Petit (1986) and Villac (2003) for more details). In this frame, and after a normalization of the length and time scale ${ }^{2}$, Hill's equations can be written as:

$$
\ddot{\boldsymbol{q}}+2 \omega \times \dot{\boldsymbol{q}}+\omega \times(\boldsymbol{\omega} \times \boldsymbol{q})=-\frac{\boldsymbol{q}}{|\boldsymbol{q}|^{3}}-\boldsymbol{q}+3\left(\boldsymbol{q}^{\mathrm{T}} \boldsymbol{a}\right) \boldsymbol{a}
$$

where $\boldsymbol{a}$ represents the unit vector from $\overline{\boldsymbol{c}}$ to the disturbing body and indicates the direction of the negative $x$-axis. The $z$-axis is taken to be along $\omega$ while the $y$-axis completes the orthogonal frame (i.e. tangent to the direction of motion of the fictitious $\overline{\boldsymbol{c}}$ ), as shown in Figure 2.

This system is Hamiltonian (autonomous) and the conservation of the Hamiltonian, expressed in position/velocity form, is known as the Jacobi integral of motion.

$$
J=\frac{|\dot{\boldsymbol{q}}|^{2}}{2}-\frac{1}{|\boldsymbol{q}|}-\frac{1}{2}\left\{3\left(\boldsymbol{q}^{\mathrm{T}} \boldsymbol{a}\right)^{2}+|\boldsymbol{\omega} \times \boldsymbol{q}|^{2}-|\boldsymbol{q}|^{2}\right\}
$$

As we know, the physical restriction $v^{2}=|\dot{\boldsymbol{q}}|^{2} \geq 0$ constrains the motion, at a given value of $J$, to a limited region of phase space, the boundary of which, known as the zero velocity surface $\mathcal{Z}$, is defined by the equation:

$$
\mathcal{Z}=\left\{\boldsymbol{q}: J+\frac{1}{|\boldsymbol{q}|}+\frac{1}{2}\left(3\left(\boldsymbol{q}^{\mathrm{T}} \boldsymbol{a}\right)^{2}+|\boldsymbol{\omega} \times \boldsymbol{q}|^{2}-|\boldsymbol{q}|^{2}\right)=0\right\}
$$

Writing $g=\frac{1}{r^{2}}\left\{3\left(\boldsymbol{q}^{\mathrm{T}} \boldsymbol{a}\right)^{2}+|\boldsymbol{\omega} \times \boldsymbol{q}|^{2}-|\boldsymbol{q}|^{2}\right\}$, where $r=|\boldsymbol{q}|, J$ is expressed as:

$$
J=\frac{v^{2}}{2}-\frac{1}{r}-\frac{1}{2} g r^{2}
$$

while $\mathcal{Z}$ is defined as the set of the real positive roots of a reduced cubic equation ${ }^{3}$ :

$$
g r^{3}+2 J r+2=0
$$

\footnotetext{
${ }^{1}$ That is, a fictitious point moving on a mean orbit (Keplerian circular orbit) around the perturbing body. The mean orbit is a circular orbit having the same mean motion, $\omega=\sqrt{\frac{\mu_{0}+\mu}{a_{0}}}$, as $c$ at an arbitrary epoch (here, $\mu_{0}$ denotes the gravitational parameter of the perturbing body and $a_{0}$ is the semi-major axis of $c$ at the chosen epoch).

${ }^{2}$ The length and time scales are defined to be $l=\left(\frac{\mu}{|\omega|^{2}}\right)^{1 / 3}$ and $\tau=\frac{1}{|\omega|}$ respectively, so that in these units, $|\boldsymbol{\omega}|=1$ and $|\boldsymbol{a}|=1$.

${ }^{3}$ See, for e.g., Conkwright (1957) for basic informations about reduced cubic equations.
} 


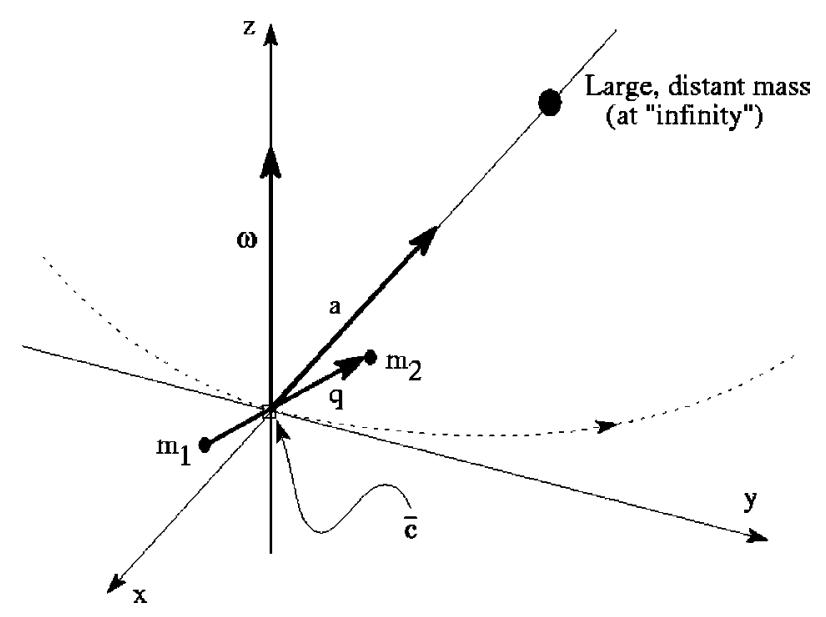

Figure 2. Geometry of Hill's problem.

The quantity $g=\left(3 x^{2}-z^{2}\right) / r^{2}$ can be expressed in terms of spherical coordinates and depends only on the longitude, $\phi$, and the latitude, $\lambda$ :

$$
g(\phi, \lambda)=3 \cos ^{2} \phi \cos ^{2} \lambda-\sin ^{2} \lambda
$$

The equations $g(\phi, \lambda)=g<0$ (resp. $g(\phi, \lambda)=g>0$ ) represent some cones with apex at the origin, axis as the $z$-axis (resp. $x$-axis) and normal section as ellipses in the $(x, y)$-plane (resp. $(y, z)$-plane). The equality $g(\phi, \lambda)=0$ parameterizes the planes $z= \pm \sqrt{3} x$.

Note that the above objects $(\mathcal{Z}$ and $J)$ are invariant with respect to the transformations $\phi \rightarrow \pi-\phi$ and $\lambda \rightarrow \pi-\lambda$, reflecting the symmetries that leave (1) invariant (time reversal reflections about the $(x, y)$ and $(y, z)$-planes, reflection about the $(x, y)$-plane and compositions). It is thus sufficient to look at these objects only in the first quadrant of the positive space $(x>0$, $y>0, z>0$ ). Now, as $J$ varies, the topology of $\mathcal{Z}$ does not change except at the critical value of the Jacobi constant, $J_{\mathrm{c}}=-\frac{3}{2} 3^{1 / 3}$. At this value of $J$, the Hill problem admits two equilibrium solutions (libration points), denoted $L_{1}$ and $L_{2}$, and located on the $x$-axis at $x_{L_{1}}=-r_{\mathrm{c}}$ and $x_{L_{2}}=r_{\mathrm{c}}$, respectively, where $r_{\mathrm{c}}=\left(\frac{1}{3}\right)^{1 / 3}$. For $J<J_{\mathrm{c}}, \mathcal{Z}$ has three disconnected components (implying a bounded regime of motion near the central body), while for $J>J_{\mathrm{c}}, \mathcal{Z}$ has now only one connected component forming two openings near the libration points and escape from the primary is now possible (see the boundary of the region of forbidden motion, i.e. shaded region in Figure 3). It can be checked in this case that the radial lines are tangent to the openings in $\mathcal{Z}$ (near the libration points) when $r=\frac{3}{2|J|}$ (see Villac, 2003). 

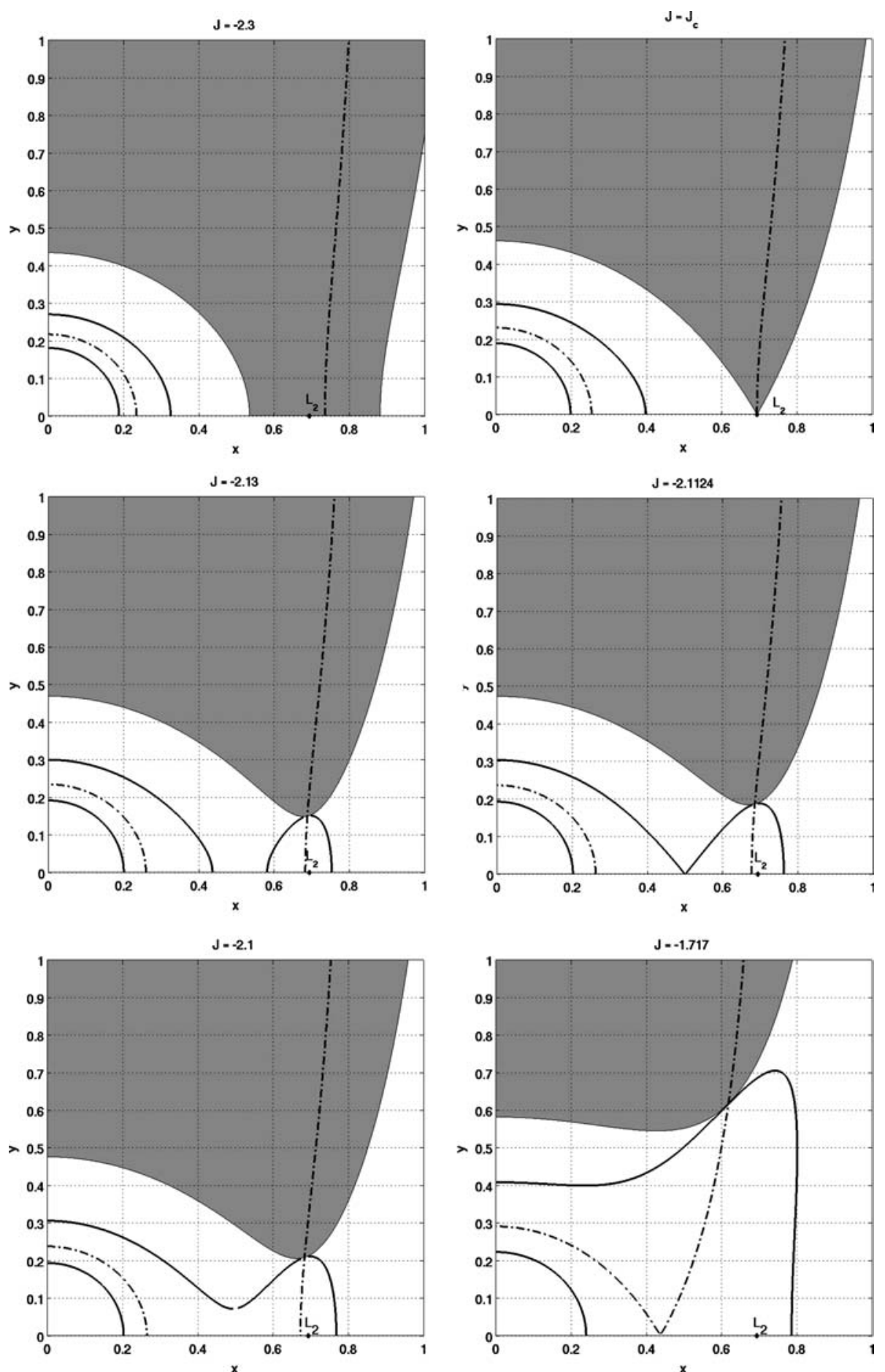

Figure 3. Limiting curves between periapsis and apoapsis in the planar Hill problem (solid line: $w(r)=0$; dash-dot line: $f(r)=0$ ). 


\subsection{DEFINITION OF PERIAPSIS}

Now, define periapses as the points of closest approach from the central body. Thus, at these points, the magnitude of the radius vector achieves a minimum and the periapsis condition can be formulated as ${ }^{4} \dot{r}=0$ and $\ddot{r}>0$.

Expressed in terms of the position vector and its derivatives, this definition is equivalent to:

$$
\left\{\begin{array}{l}
\boldsymbol{q}^{\mathrm{T}} \dot{\boldsymbol{q}}=0 \text { and } \boldsymbol{q} \neq 0 \\
v^{2}+\boldsymbol{q}^{\mathrm{T}} \ddot{\boldsymbol{q}}>0
\end{array}\right.
$$

where $\ddot{\boldsymbol{q}}$ represents the acceleration of the particle in the rotating frame as expressed in (1).

Thus we can see that periapsis is defined by a geometrical condition ( $\boldsymbol{q}$ orthogonal to $\dot{\boldsymbol{q}}$ ) plus a condition on the speed of the particle. In fact, the geometrical condition (apsis condition) projects onto the whole Hill region (region of allowable motion delimited by $\mathcal{Z}$ ), while the restriction imposed by the periapsis condition is more restrictive, as we will see in the next section. Note that when $v^{2}+\boldsymbol{q} \cdot \ddot{\boldsymbol{q}}<0$, the magnitude of the radius vector achieves a maximum. At such points, the particle is said to be at apoapsis.

Even though the definition of $\dot{\boldsymbol{q}}$ and $\ddot{\boldsymbol{q}}$ are different in the rotating and the inertial frames (having the same origin), it can be easily checked that the notion of periapsis (or apoapsis) is independent of the frame. That is:

$$
\begin{cases}\left(\boldsymbol{q}^{\mathrm{T}} \dot{\boldsymbol{q}}\right)_{\mathrm{I}} & =\left(\boldsymbol{q}^{\mathrm{T}} \dot{\boldsymbol{q}}\right)_{\mathrm{R}} \\ \left(v^{2}+\boldsymbol{q}^{\mathrm{T}} \ddot{\boldsymbol{q}}\right)_{\mathrm{I}} & =\left(v^{2}+\boldsymbol{q}^{\mathrm{T}} \ddot{\boldsymbol{q}}\right)_{\mathrm{R}}\end{cases}
$$

where the subscripts I and $\mathrm{R}$ refer, respectively, to an inertial and a rotating frame centered at $\overline{\boldsymbol{c}}$.

The notion of periapsis (or apoapsis) does depend, however, on the force field considered. For example, the notion as defined in the Hill problem and the Kepler problem are not equivalent.

For the Kepler problem in a rotating frame, we have

$$
\ddot{\boldsymbol{q}}=-2 \boldsymbol{\omega} \times \dot{\boldsymbol{q}}-\boldsymbol{\omega} \times(\boldsymbol{\omega} \times \boldsymbol{q})-\frac{\boldsymbol{q}}{|\boldsymbol{q}|^{3}},
$$

so that periapsis is achieved when $\boldsymbol{q}$ and $\dot{\boldsymbol{q}}$ are orthogonal and

$$
v^{2}>\frac{1}{r}-2 \omega^{\mathrm{T}}(\boldsymbol{q} \times \dot{\boldsymbol{q}})-|\boldsymbol{\omega} \times \boldsymbol{q}|^{2} .
$$

The acceleration in the Hill problem differs from that of the Kepler problem in a rotating frame by the term $-\boldsymbol{q}+3\left(\boldsymbol{a}^{\mathrm{T}} \boldsymbol{q}\right) \boldsymbol{a}$. That is the periapsis condition is given by:

\footnotetext{
${ }^{4}$ Strictly speaking, one can also have $\ddot{r}=0$ at periapsis but we will restrict ourselves to the
} definition given. 


$$
v^{2}>\frac{1}{r}-2 \omega^{\mathrm{T}}(\boldsymbol{q} \times \dot{\boldsymbol{q}})-|\boldsymbol{\omega} \times \boldsymbol{q}|^{2}+|\boldsymbol{q}|^{2}-3\left(\boldsymbol{a}^{\mathrm{T}} \boldsymbol{q}\right)^{2}
$$

Thus, for $|\boldsymbol{q}|^{2}-3\left(\boldsymbol{a}^{\mathrm{T}} \boldsymbol{q}\right)^{2}>0$, a particle can be at periapsis in the Hill problem while being at apoapsis in the Kepler problem. For $|\boldsymbol{q}|^{2}-3\left(\boldsymbol{a}^{\mathrm{T}} \boldsymbol{q}\right)^{2}<0$, the situation is opposite, that is, a particle can be at apoapsis in the Hill problem while being at periapsis in the Kepler problem. These cases can in fact occur only for small eccentricities, i.e., nearly circular orbits.

Finally, one can notice that the notion of periapsis and apoapsis agree in both problems when $|\boldsymbol{q}|^{2}-3\left(\boldsymbol{a}^{\mathrm{T}} \boldsymbol{q}\right)^{2}=0$, which corresponds to a cone in position space with apex at the origin, axis given by the $x$-axis and with normal section corresponding to circles in the $(y, z)$ coordinates. The first situation $\left(|\boldsymbol{q}|^{2}-3\left(\boldsymbol{a}^{\mathrm{T}} \boldsymbol{q}\right)^{2}>0\right)$ occurs 'outside' the cone while the other case $\left(|\boldsymbol{q}|^{2}-3\left(\boldsymbol{a}^{\mathrm{T}} \boldsymbol{q}\right)^{2}<0\right)$ occurs 'inside' the cone (i.e., the region containing the axis).

In the remainder, our focus will be on the case of the Hill problem and periapsis will thus be defined by (9).

\section{Partitioning of Position Space}

An interesting fact in the Hill problem (this also holds for the restricted three body problem) is that the periapsis condition (resp. apoapsis condition) reduces to a condition independent of the velocity in the planar case. Indeed, one can use the Jacobi constant (4) to express the speed of the particle in terms of its position and energy. This will appear clearly in Section 3.2.

First, we re-express the condition involving the acceleration in the definition of an apsis by using the equations of motion (1):

$$
\boldsymbol{q}^{\mathrm{T}} \ddot{\boldsymbol{q}}=2 \boldsymbol{\omega}^{\mathrm{T}}(\boldsymbol{q} \times \dot{\boldsymbol{q}})+|\boldsymbol{\omega} \times \boldsymbol{q}|^{2}-\frac{1}{r}-r^{2}+3\left(\boldsymbol{q}^{\mathrm{T}} \boldsymbol{a}\right)^{2}
$$

or, using the previously defined quantity $g$ :

$$
\boldsymbol{q}^{\mathrm{T}} \ddot{\boldsymbol{q}}=2 \boldsymbol{\omega}^{\mathrm{T}}(\boldsymbol{q} \times \dot{\boldsymbol{q}})+g r^{2}-\frac{1}{r}
$$

Then, at any apsis in the planar case, one can readily check that $\omega^{\mathrm{T}}(\boldsymbol{q} \times \dot{\boldsymbol{q}})= \pm r v$ (the plus sign being associated with direct motion and the minus sign with retrograde motion), so that the periapsis condition $v^{2}+\boldsymbol{q}^{\mathrm{T}} \ddot{\boldsymbol{q}}>0$ can be re-expressed ${ }^{5}$ in the direct case, as:

$$
2 r^{2} v+f(r)>0
$$

where the function $f(r)$ is given by:

$$
f(r)=2 g(\phi, \lambda) r^{3}+2 J r+1 .
$$

${ }^{5}$ By multiplying this condition by $r$ and using the Jacobi constant as given in Equation (4). 
TABLE I

Constraints on periapsis and apoapsis in the planar Hill's problem

\begin{tabular}{lll}
\hline & Direct case & Retrograde case \\
\hline Preiapsis & $2 r^{2} v+f(r)>0$ & $f(r)-2 r^{2} v>0$ \\
Apoapsis & $2 r^{2} v+f(r)<0$ & $f(r)-2 r^{2} v<0$ \\
\hline
\end{tabular}

The other apsis conditions are derived in a similar manner, the results being presented in Table I. Note that the cases of direct apoapsis and retrograde periapsis are only possible if $f(r)<0$ and $f(r)>0$, respectively. Thus, in the region where $f(r)>0$ (close to the center of the frame), a particle cannot be at direct apoapsis, while in the regions where $f(r)<0$, a particle cannot be at a retrograde periapsis (for a fixed value of $J$ ).

In the spatial problem, $\boldsymbol{\omega}^{\mathrm{T}}(\boldsymbol{q} \times \dot{\boldsymbol{q}})=r v \cos \theta$ where $\theta$ represents the inclination as defined in the rotating frame. The above results apply to this case by replacing the direct and retrograde cases with $\theta$ below or above $90^{\circ}$. The limits between regions with only periapsis or apoapsis possible are independent of $\theta$ (we have indeed $-1 \leq \cos \theta \leq 1$ ) and are thus given by the conditions presented in Table I. Therefore, the discussion below applies to the spatial problem.

\subsection{SCALING OF THE ZERO VELOCITY SURFACE}

The equation $f(r)=0$ is a cubic in $r$, very similar to the one defining the zero velocity surface, and can be solved explicitly. In fact, this equation is the equation defining $\mathcal{Z}$, modulo a scaling of the radius and the Jacobi constant. More precisely, letting $r=4^{-1 / 3} \bar{r}$ and $J=\frac{1}{2} 4^{1 / 3} \bar{J}$, Equation (13) transforms into (5). Thus, all the results applying to the case of the zero velocity surface apply here, modulo the above scaling.

In particular, the surface $f(r)=0$ determines a bounded region close to the primary where $f(r)>0$ and no direct apoapsis is possible. Also, far from the primary, we again have $f(r)>0$ and no direct apoapsis is possible. This means that at a point where $\boldsymbol{q}$ and $\dot{\boldsymbol{q}}$ are orthogonal and $\boldsymbol{\omega}^{\mathrm{T}}(\boldsymbol{q} \times \dot{\boldsymbol{q}})>0$, a particle will locally move away from the primary.

In fact, these regions connect when the surface opens at the value $\bar{J}=J_{\mathrm{c}}$. In non-scaled units, this corresponds to a value of Jacobi constant equal to $J=-\frac{3}{2}\left(\frac{3}{2}\right)^{1 / 3}$. At this value of $J$, all trajectories with $y=0$ and $\dot{x}=0$ will move away from the primary when $\dot{y}>0$ on the positive $x$-axis and $\dot{y}<0$ on the negative $x$-axis. For many trajectories, this will mean escape.

Similarly, outside the region $f(r)>0$, the motion is constrained so that no retrograde periapsis is possible. It can be easily proven that such regions always exist for $J<0$. In fact, all points on $\mathcal{Z}$ correspond to such a situation 
when $r<-\frac{3}{2 J}$. Recall that this value of $r$ corresponds to the tangency of $\mathcal{Z}$ with the radial lines when $J \geq J_{\mathrm{c}}$ and lies in the region of forbidden motion for $J<J_{\mathrm{c}}$. Thus, for $J<J_{\mathrm{c}}$, all points on $\mathcal{Z}$ in the inner region correspond to apoapsis, while all points of $\mathcal{Z}$ in the outer region correspond to periapsis. When $J \geq J_{\mathrm{c}}$, the situation holds equally well, ${ }^{6}$ if the inner region is determined by $r<-\frac{3}{2 J}$. In fact, this result shows that the outer components of $f(r)=0$ meet $\mathcal{Z}$ at $r=-\frac{3}{2 J}$ and thus crosses the $x$-axis at $r_{\mathrm{c}}$ when $J=J_{\mathrm{c}}$.

Finally, we should note that when $J<J_{\mathrm{c}}$, the inner component of $f(r)=0$ lies about midway between the origin and $\mathcal{Z}$ and exactly midway on the $y$ axis, i.e., at $r=-\frac{1}{2 J}$, similarly as in the two body problem.

Figure 3 illustrates these situations in the first quadrant of the $(x, y)$-plane (the other quadrants being obtained by symmetries).

\subsection{SEPARATION BETWEEN PERIAPSIS AND APOAPSIS REGIONS}

Even though the above discussion gives some constraints on the periapsis and apoapsis regions, the results can be strengthened by looking at the signs (and hence the roots) of the functions $f(r)-2 r^{2} v$ and $f(r)+2 r^{2} v$, where $v$ is expressed in terms of position and the Jacobi constant. These functions involve square roots and the zeros cannot be solved explicitly. These zeros can, however, be easily computed numerically. In fact, these zeros are the roots of the sixth degree polynomial $w(r)=\left(f(r)-2 r^{2} v\right)\left(f(r)+2 r^{2} v\right)$ :

$$
w(r)=4(g-1) r^{3}\left(g r^{3}+2 J r+1\right)-4 r^{3}+(2 J r+1)^{2}
$$

The roots of $w(r)$ can be sorted according to the sign of $f(r)$ so as to make them correspond to the zeros of the above functions. Therefore, with the help of Table I, one obtains the following diagram for the partitioning of a radial line in the Hill region (assuming the roots exist) between periapsis and apoapsis regions:

(1)

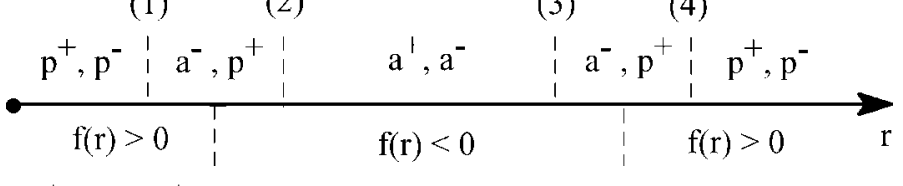

where $a^{+}, a^{-}, p^{+}$and $p^{-}$denote, respectively, direct and retrograde apoapsis and periapsis, and the numbers (1)-(4) represent the roots of $w(r)$.

Thus, close to the primary, only periapses are possible and, close to the surface $f(r)=0$, only retrograde apoapses and direct periapses are possible.

\footnotetext{
${ }^{6}$ These facts can be proven directly from the reduced cubic equations: on $\mathcal{Z}, v=0$ and periapses (apoapses) are defined as $f(r)>0(f(r)<0)$. Also we have the relation $g r^{3}+2 J r+2=0$, so that $f(r)=J r+\frac{3}{2}$. The result follows.
} 
More generally, looking at Figure 3 where the roots of $w(r)$ have been plotted (thick solid lines), one can obtain an idea of what these regions look like.

Further properties of the roots of $w(r)$ can be obtained by looking at $w$ as a function of $J$. Indeed, $w(r)$ is a quadratic form in $J$, so that even though the roots of $w(r)$ cannot be solved a priori by radicals, one can solve for $J$ as a function of $r$.

$$
w(J)=4 r^{2} J^{2}+4 r\left(1+2(g-1) r^{3}\right) J+\left[1+4(g-2) r^{3}+4 g(g-1) r^{6}\right]
$$

The reduced discriminant of $w(J)=0$ is $\Delta^{\prime}=16 r^{5}\left[1-(g-1) r^{3}\right]$, so that, in particular, $\Delta^{\prime}>0$ as soon as $g \leq 1$ (regions containing the $z$-axis) or $r<(g-1)^{-1 / 3}$ when $g>1$ (openings regions). Thus, for $g>1, w(r)>0$ for $r>(g-1)^{-1 / 3}$ and only periapses are possible. The value of $r=(g-1)^{-1 / 3}$ is actually reached for $J=-\frac{3}{2}(g-1)^{\frac{1}{3}}$. In the particular case of the $x$-axis, the corresponding values of $r$ and $J$ are, respectively, $r=2^{-1 / 3} \simeq 0.793$ and $J=-\frac{3}{2} 2^{1 / 3} \simeq-1.889$.

From (15), one can also see that for each value of $r$, there corresponds two values of $J\left(J^{+}\right.$and $\left.J^{-}\right)$for which $w(J)=0$. This fact allows us to show that the points at which the roots (2) and (3) in the previous diagram collapse as $J$ varies (if they exist) also correspond to the points at which the roots (1) and (4) must collapse and the corresponding values of $J$ are $J^{-}$and $J^{+}$, respectively. Notably, $w(r)>0$ for any value of $r$ on a radial line defined by $g>1$, when $J>-\frac{3}{4}[4(g-1)]^{1 / 3}+\sqrt{\frac{3}{4} \frac{1}{[4(g-1)]^{1 / 3}}}$. The last root on this radial line will occur when $J$ equals this critical value, at a radius of $[4(g-1)]^{1 / 3}$. In the particular case of the $x$-axis, these critical values of collapse and disappearance of roots are $r=0.5$ and $J=-\frac{3}{2}-\frac{1}{2} \sqrt{\frac{3}{2}} \simeq-2.1124$ and $J=-\frac{3}{2}+$ $\frac{1}{2} \sqrt{\frac{3}{2}} \simeq-0.8876$, respectively. This is in concordance with the middle right plot shown in Figure 3.

\section{Applications}

In this section two applications of the previous results to the dynamics in the Hill problem are presented: a proposed definition for the libration point regions and the asymptotic variations of quasi-circular orbits.

\subsection{LOCAL ESCAPE AND CAPTURE AT LOW ENERGIES}

We have seen in Section 3.1 that the formulation of periapsis allowed us to directly obtain the local behavior on the zero velocity surface. Points on $\mathcal{Z}$ 
for which $r<-\frac{3}{2 J}$ (recall that $J<0$ here) correspond to apoapses (that is trajectories locally fall towards the origin), while the points with $r>-\frac{3}{2 J}$ correspond to periapsis (trajectories locally get farther from the origin). For $J \leq J_{\mathrm{c}}$, the sphere $r=-\frac{3}{2 J}$ is contained in the region of forbidden motion and thus separate the inner and outer Hill's regions. When $J>J_{\mathrm{c}}$, we know from the linearized dynamics around the libration points (see Gómez et al., 2001 for example) that periodic and quasi-periodic orbits that stay in the vicinity of the libration points exist (center manifold). These trajectories belong neither to the inner Hill's region nor to the exterior region and a third region, the 'neck' region, naturally appears as a feature of the dynamics. In the linearize theory, these neck regions are defined as the regions bounded by $\mathcal{Z}$ and two planes surrounding the center manifold (see Gómez et al., 2001 for example), but the separation between the inner, neck and outer regions remain rather arbitrary.

For $J<-2.1124$, the previous results allow us to give a natural definition of the neck regions in the non-linear setting and a bound to the minimum distance from the primary that a non-transit trajectory ${ }^{7}$ coming from infinity can reach.

Indeed, looking at the middle left plot of Figure 3, we can see that the third and fourth roots of $w(r)$ delineate a closed region near the libration points that closes on $\mathcal{Z}$ at $r=-\frac{3}{2 J}$. In this region, only direct periapsis and retrograde apoapsis are possible. We thus define this close region as the neck region. The inner region is then the region containing the origin and bounded by $\mathcal{Z}$ and this neck region, while the outer region is the complementary of these two regions.

This definition naturally extends the definition for $J \leq J_{\mathrm{c}}$, as well as the change of behavior of the trajectories meeting $\mathcal{Z}$ at $r=-\frac{3}{2 J}$. This definition allows us also to define local escape and capture as the trajectories that cross the outer and inner boundary of the neck region, respectively.

Indeed, a trajectory crossing the outer boundary of the neck region towards the exterior region locally moves away form the origin and, thus, it must pass by an apoapsis before returning towards the neck region. However, in the exterior region surrounding the neck region, only periapses are possible, thus making a quick return impossible (retrograde apoapsis regions in the exterior Hill's region exist but are farther than $r=2$ at the energies considered. Direct apoapsis regions do not exists). Therefore, these trajectories are defined as locally escaping.

Similarly, trajectories crossing the inner boundary of the neck region towards the origin must pass through periapsis before begin able to come back

\footnotetext{
${ }^{7}$ Non-transit trajectories come from the inner or outer regions, enter the neck region and return to the same region from which they originate.
} 
towards the neck region again. This means that these trajectories must have a close encounter with the origin and are thus defined as locally captured trajectories. This discussion does not prove that the locally captured trajectories must perform a revolution around the origin. However, for the low value of energies considered, numerical integrations show that this is indeed the case (see Villac and Scheeres, 2003).

Numerically these definitions allow us to quickly decide if a trajectory escape (or is captured) without having to integrate to an arbitrary radius. Indeed, checking the sign of $f(r)$ and $w(r)$ suffices to decide of these issues.

\subsection{HILL'S PERIODIC ORBITS AND QUASI-CIRCULAR TRAJECTORIES}

Another interesting consequence of this partitioning of the position space is that for low values of the Jacobi constant, the two inner roots of $w(r)$ define the location of quasi-circular orbits. Indeed, the surfaces defined by the two inner roots of $w(r)$ are almost circular (they do not depend on $g$ to the first order in their dependence on $J$ ). The periapsis of quasi-circular orbits must lie below the first curve in the retrograde case and its apoapsis must lie above it. Since the eccentricity is small at each point of these orbits, the two apses must be close together, and close to the inner surface of $w(r)=0$. The same situation holds true in the direct case with the second curve.

It can be easily checked that variations of these two inner surfaces with $J$ is given by:

$$
r^{ \pm}=-\frac{1}{2 J} \pm \frac{1}{2 \sqrt{2} J^{5 / 2}}+\mathcal{O}\left(\frac{1}{J^{3}}\right)
$$

where the plus sign refers to the most inner surface.

Thus, when $J \rightarrow-\infty$, the two inner surface $w(r)$ collapse towards a single sphere $r=-\frac{1}{2 J}$ and the distinction between retrograde and direct motion disappears, in concordance with the two body problem results discussed in the introduction (as $J \rightarrow-\infty$, the bounded regime of motion in Hill's problem approaches the Keplerian dynamics since $\mathcal{Z}$ shrinks to zero and close to the origin the perturbations becomes negligible as compared to the Keplerian terms). Moreover, these results give us an asymptotic expression for the separation between direct and retrograde motion. Indeed the first and second roots of $w(r)$ separate as $\frac{1}{\sqrt{2} J^{5 / 2}}$.

Finally, note that for these low values of energy, the Hill's periodic orbits (families $f$ and $g$ in Hénon's terminology) become quasi-circular (see Hénon, 1969) and thus follow the above results. We can also note that the retrograde orbits which are more stable than the direct ones are constrained to be closer to the origin, as our physical intuition would suggest. 
Thus, we see that the three roles played by the circular orbits in the two body problem (as discussed in Section 1) are now played by several objects, but stay closely related for low values of the Jacobi constant. In fact, only the geometrical role of the circular orbits (scaling of the zero velocity surface) can be continued into the Hill's problem as a single, connected object ${ }^{8}$ (defined by the inner root of $f(r)=0$ ), while the dynamical roles of the circular orbits result in different curves/surfaces according to the direction of motion (direct or retrograde). The circular periodic orbits are continued into the distinct families, $f$ and $g$ (in the planar case) and the separation between periapsis and apoapsis regions are now played by the curves $w(r)=0$, one curve delimiting between direct periapsis and direct apoapsis while a different curve delimits retrograde periapsis from retrograde apoapsis. The surface $f(r)=0$ plays the role of a mean separatrix from which the curves characterizing direct and retrograde motion separate.

\section{Conclusion}

While not necessitating more sophisticated mathematics than the analysis of the zero velocity surface, $\mathcal{Z}$, the analysis of the concept of apsis yields deeper qualitative properties of the dynamics of the Hill's problem than does the simple study of $\mathcal{Z}$. It gives a direct physical insight into the global dynamics of the problem with limited computational effort and may thus represent a useful approach to these interesting non-linear dynamics, complementary to quantitative tools such as the analytical/numerical continuation of periodic orbits or the study of the libration point dynamics. Hill's problem also shares a common realm of application with the circular restricted three body problem, thus similar computations to the one presented in this article are applicable to this problem.

\section{References}

Conkwright, N.B.: 1957, Introduction to the Theory of Equations, Ginn, Boston.

Gómez, G., Koon, W.S., Lo, M.W., Marsden, I.E., Masdemont, J., and Ross, S.D.: 2001, 'Invariant manifolds, the spatial three-body problem and space mission design', the Proceedings of AIAA/AAS Astrodynamics Specialist Meeting, Quebec City, Quebec, Canada, August 2001.

Hénon, M.: 1969, 'Numerical exploration of the Restricted Problem. V. Hill's case: Periodic Orbits and Their Stability', Astr. Astrophys. 1, 223-238.

Hénon, M. and Petit, J.M.: 1986, 'Series expansions for encounter-type solutions of Hill's problem', Celest. Mech. Dyn. Astr. 38(1) 67-100.

${ }^{8} \mathrm{At}$ least, if one is only concerned with the dynamics close to the primary. 
Hill, G.W.: 1878, 'Researches in the Lunar theory', Amer. J. Math. 1, 5-26.

Szebehely, V.: 1967, Theory of Orbits, the Restricted Problem of Three Bodies, Academic Press, New York.

Villac, B.F.: 2003, Dynamics in the Hill problem with applications to spacecraft maneuvers, Ph.D thesis, The University of Michigan.

Villac, B.F. and Scheeres, D.J.: 2003, 'Escaping trajectories in the Hill three-body problem and applications', J. Guidance Control Dyn. 26 (2) 224-232. 